correct management of both maladies the results will not give hope and encouragement to sufferers or their medical advisers.

The tuberculosis service in this country may have reached as high a peak of perfection as it has ever attained since its inauguration, but this will hardly avail the diabetic unless his metabolic disturbance be at the same time under expert supervision. Experience has shown that good treatment of the pulmonary disease with inadequate supervision of the diabetes is as harmful as poor treatment of the phthisis with proper control of the diabetes. It goes without saying that bad treatment of both conditions is inevitably disastrous. The truth is that in many instances the tuberculous diabetic is uncared for according to the best medical standards, and the evil effects of this are brought to our notice in the published results of treatment.

At the few clinics where adequate control of both diseases has been enforced from the beginning the results have indicated that the outlook for the diabetic who develops phthisis hardly differs from that of the non-diabetic. Figures published from sanatoria in the past have led to false conclusions as to the seriousness of the combination of these two diseases; the bad results obtained were probably due to inadequate control of both conditions according to modern therapeutic standards. Unfortunately this fact has not been fully realized, with the result that the tuberculous diabetic has acquired a stigma of hopelessness based on mismanagement of his diseases.

Publication of the new version of Section I of the Appendix to Memorandum 37/T (Revised), which has been accepted by the Ministry of Health (Supplement, May 31, p. 111), raises important considerations concerning the tuberculous diabetic. In the section on the classification of patients suffering from tuberculosis, it is stated that "all cases with grave complications, whether they are tuberculous or not, should be classified in this group [group B3] (e.g., diabetes, tuberculosis of intestine or larynx)." My first objection to this new classification is that it places the diabetic whose disease is complicated by a small pulmonary lesion in the same category as the patient with advanced bilateral fibro-cavernous disease. Secondly, the presence of diabetes is looked upon in the same light as tuberculous enteritis or laryngitis. Here, surely, a gross injustice is done to the diabetic. Whereas the onset of tuberculous enteritis in most cases heralds a fatal issue, the outlook for the tuberculous diabetic, as emphasized already, is never as serious as this unless by virtue of the extent of his pulmonary disease. The combination of diabetes and tuberculosis should never be regarded as the equivalent of hopeless and irrecoverable disease.

Apart from the objections on medical grounds to placing tuberculous diabetics in the class B3 one can imagine many othęr disadvantages that such a patient may suffer owing to his consideration in this group. B3 cases have always been regarded as suffering from advanced pulmonary disease, so that there has been no urgency in considering their admission to sanatoria for treatment. They may be left at home, if conditions allow, or they may be admitted to homes or hospitals for advanced cases. It would be most unfortunate if tuberculosis officers should be led, after reading the new classification, to treat their tuberculous diabetics in a like manner. The development of phthisis as a complication of diabetes should be looked upon as a medical emergency, requiring prompt treatment for both conditions. An unnecessarily long wait for admission can only mean advance of the pulmonary disease together with inadequate control of the diabetes and a poor outlook for the patient; accordingly, such patients warrant priority on the waiting-lists. Finally, are the benefits of the maintenance grants from the Treasury (Memo 266/T) to be withheld from tuberculous diabetics under the new scheme of classification?

The diabetic surely has to suffer enough already on account of his unfortunate affliction without having additional difficulties put in his way which are based merely on prejudice and receive no support from properly instituted clinical studies. It is to be most earnestly hoped that further consideration may be given to the amended Section I, wherein diabetics are included in class B3, and that special units will soon be established for the treatment of tuberculous diabetics.-I am, etc.,

\section{Classification of Tuberculous Patients}

SIR,-Dr. H. E. Stevens's letter (May 31, p. 782) draws attention to a deficiency in the new classification of tuberculosis terms recently adopted by the Ministry of Health. The object of adopting the new classification is not stated, but presumably it is mainly to secure increased accuracy of returns through clarification of the headings used in compiling them. My own belief is that this object will not be attained by the present proposals, and if other tuberculosis workers feel the same they might consider whether it is worth their while to ask for a re-examination of the scheme now put forward.

The following are some of the grounds on which I base my opinion :

(1) The replacement of the self-explanatory terms "minus" and "plus" by A and B is to me a retrograde step, and is bound to lead to mistakes in classification owing to the absence of any apparent relation of the letters $\mathrm{A}$ and $\mathrm{B}$ with either positive or negative cases.

(2) The definitions of respiratory and non-respiratory tuberculosis are not free from criticism. One expects these definitions to be helpful to the extent that few, if any, occasions will arise when it will be impossible to classify a patient by their use. But such occasions are bound to happen when no provision is made in the definitions for including conditions other than those specified-for example, endo-bronchial tuberculosis. The difficulty would be overcome by substituting the words "a respiratory case includes ..." for the words " a respiratory case should be. ..."

(3) A "combined" case-e.g., with pulmonary and glandular tuberculosis-is classified as a pulmonary case, as at present. But if such a patient is found to have tubercle bacilli in his excised glands he will be classified as $B$, giving the impression that he is infectious in the same degree as a patient who is discharging tubercle bacilli in his sputum. The definition of an infectious pulmonary case is incomplete, like the general definition of a pulmonary case. The finding of tubercle bacilli should be more definitely related to the pulmonary lesion by specifying the situations where the bacilli will be regarded as evidence of their pulmonary origin.

(4) The definition of a class B case suffers from the further defect that it makes no provision for reclassifying a recovered case who comes on the dispensary register again after a period of years. According to the literal application of the wording, a person who had tuberculous cervical glands in childhood with tubercle bacilli in the pus from the glands, and who comes on the register with any tuberculous condition 20 years later, is to be called a $B$ case. For the purposes of compiling uniform statistics this procedure may be very desirable, but the statistics themselves can hardly be helpful. I submit that a definition of a "return" or "restored" case is required, and that the classification of such a case should be based on the current findings and not on the bacteriological results obtained many years previously.

(5) The subsections of paragraph III of the memorandum are inconsistent. Subsection (2) states that respiratory A.s and B.s should be subdivided; subsection (3) states that they should be further subdivided. Yet there is only one basis of subdivision described-viz., into groups 1,2 , and 3 . The meaning is clear enough, but the wording is confusing.

(6) The definition of "quiescent" does not make any allowance for variations in bacteriological control by different observers: some will rely on repeated slide examinations, some will prefer culture or inoculation methods. But, according to the definition, unless three consecutive monthly slides are negative, the case does not fulfil the criteria required for quiescence. Moreover the definition does not take account of the fact that a patient may not be able to produce sputum when required at monthly intervals, and it seems clear that it cannot be strictly observed as it stands at present.

(7) The classification "arrested" does not seem to serve any useful purpose. In practice it is never used to describe any patient, and rather than set down details for its interpretation I consider it should be abolished.

(8) Does the definition of a "recovered" case apply only to pulmonary patients? If it is intended to apply to nonpulmonary patients also, then it should be stated, as at present a non-pulmonary case can be written off as "recovered" after three years.

(9) Regarding the definition of "active case," I consider it unfortunate that, by implication, all patients not positive within the preceding three months may be taken to be classifiable as not active-i.e., quiescent. I am sure this is not the intention, but the wording is not so helpful as it might be.

(10) The use of the word "stationary" to denote a case in which the patient remains in statu quo appears unscientific and unnecessary, for if a patient was "quiescent" at the beginning 
of the "stationary" period, he may continue to be described as quiescent rather than as stationary.

(11) Among the definitions not mentioned is the Ministry's own term "inactive" as used in the classification of mass radiography findings. I think it a pity that the opportunity has not been taken to make clear the implications of the use of this term.

(12) The inclusion of directions for the medical assessment of patients suffering from pleurisy has the appearance of an intrusion in a document dealing with the classification of patients suffering from tuberculosis. I consider that the direction to regard pleurisy as tuberculous unless proved otherwise is inconsistent with the facts $(a)$ that less than half of the patients who suffer from pleural effusion ultimately develop pulmonary tuberculosis, and $(b)$ that pleural effusion is frequently part of a primary tuberculous process. The requirement that cases of pleural effusion should be regarded, and presumably notified, as tuberculous will have repercussions in the matter of the notification of primary tuberculosis in general.

(13) The new Non-Respiratory classification will not show the part affected. The old classification was in four groups: (1) bones and joints, (2) abdominal, (3) other organs, (4) peripheral glands. It is desirable that the subdivisions of the old classification be incorporated in the new groupings.

(14) If the old method of classification is to be amended, let us make sure it is soundly based, because it will involve the reclassification of all the cases on the dispensary registers so as to afford comparison with previous years.

In issuing their memorandum the Ministry have no doubt acted on the advice of the Joint Tuberculosis Council, but it is regrettable that no opportunity was given to tuberculosis workers in general to express an opinion before the recommendations were forwarded to the Ministry.-I am, etc.,

F. C. S. BRADBURY

Preston. Central Consultant Tuberculosis Officer.

\section{Artificial Insemination}

SIR,-There can be few subjects which call forth more emotional (as opposed to intellectual) judgments than A.I.D. The letters from Dr. Eustace Chesser (May 24, p. 738) and Dr. Farquhar Murray (June 7, p. 826) are cases in point. The latter is so carried away by his disgust of the whole procedure that he does not present the facts quite as they are. The only donors I have ever met are unselfish and intelligent men who do not sell or barter their spermatozoa but who agree to give them, as they would blood for transfusion, to help those in need-i.e., equally unselfish and intelligent couples who, because of the husband's complete sterility, have been deprived of begetting, bearing, and rearing the children they earnestly desire, more often than not through no fault of their own. Dr. Chesser, on his part, paints a somewhat lurid picture of the formidable hazards surrounding those rash enough to indulge in A.I.D. He says he has abandoned the practice but cmits the facts and figures that induced him to do so. For example, he does not tell us how many cases he has dealt with by A.I.D., nor how old the resulting children are ; neither does he give any indication as to what proportion of his cases has ended in psychological disaster; he seems to infer that there are few, if any, good results. Possibly facts and figures are lacking and he has arrived at his conclusions on theoretical considerations only. His letter wculd have carried more conviction had he also painted the other half of the picture, which undoubtedly exists.

The marriage in danger of being wrecked by the smouldering resentment of the maternally inclined woman against her sterile mate and the profound sense of failure and regret which must be felt by any but the most insensitive husband: in my experience this is the situation which, unless resolved, besets two people with "formidable hazards." There are of course a number of ways out, and it lies within the doctor's sphere to try honestly and without prejudice or emotional bias to help such couples, should they consult him, to find their particular solution. The possibilities are: to be sufficient unto themselves and to sublimate in various directions; to part and start afresh; to adopt; or, finally, to secure a child or children by A.I.D. A small proportion may after careful thought choose this last, the husband conscious of his wife's strong desire to bear a child and preferring to fend for one that is at least hers rather than one that comes of neither of them. His sense of inferiority and of guilt tends to remain while the cradle is empty and is not, in my experience, replaced by jealousy of the child but by great tenderness towards it and towards a wife who has, in the sense that matters to him, kept faith ; while she in her turn feels a lasting gratitude towards her husband for his generosity in allowing A.I.D. So far I have not met the unhappy situation Dr. Chesser so vividly depicts ; quite possibly I have not dealt with so many cases as he has and my experience of the outcome of A.I.D. is therefore more limited than his. His warning will ensure a still more careful selection of cases. Finally I would suggest that it is early days to pass sweeping judgments in either direction. It is well to bear in mind that the demand for A.I.D., no less than for control of fertility, has come from the lay public. It is of prime importance that doctors should think about and weigh these things in the light of their special knowledge and experience, setting aside in so far as they can, or at any rate recognizing, their own particular emotional bias in the matter.-I am, etc.,

Crediton, Devon.

Margaret Hadley Jackson.

\section{Acid Drinks and Sulphonamide Therapy}

SIR,- It is pertinent to ask the advocates of freely drinking of the fruit juice beverages by what processes the vegetable acids or their salts, citrates, etc., come to be passed off in urine so as not to increase its acidity. The acid part gets oxidized and the other parts also get oxidized, $\mathrm{CO}_{2}$ and $\mathrm{KHO}$ or $\mathrm{H}_{2} \mathrm{O}$ being end-results. The amount of oxygen required to achieve this end is not negligible. The citric acid molecule for its change must have added to it 9 molecules of oxygen; its molecular weight, given as 210.1 , requires of oxygen $9 \times 16$, or 144. Where and how is the citrate to get it?

The bodily state in each case must be considered in regard to this important matter. It is of course the doctor's privilege to deal with this. The sulphonamides themselves do make some demands upon the respiratory functions, all of whose details, including of course the R.B.C.'s, may be affected; and in many cases the disease may be one directly affecting respiration. My colleagues in the eye specialty need not be told that prolonged or drastic degradation of oxygen supply and possibly the presentation of un- or imperfectly de-toxicated products is of importance to the retina and the lens. Others should be told.

The drinking of large quantities of water may be rather relished as an uncommon item, and if taken cold or even chilled or iced may, if thought advisable, send more of it to the kidney rather than the skin or lung for elimination. A lump of sugar in it used to be liked and would be welcome as a variant Lemon and orange juice drinks are taken far too little diluted in many cases, and it might be wise to prescribe the actual diluition considered safe as well as the total daily allowance. In the sick there are limits to the capacity for dealing with excess in $\mathrm{H}_{2} \mathrm{O}$ and $\mathrm{CO}_{2}$, methaemoglobin, and the disease products and those due to our drugs. The wisdom of the ancients is still apropos: Est modus in rebus! sunt certi denique fines. Quos ultra citraque nequit consistere rectum.-I am, etc.,

$$
\text { Giasgow. }
$$

ED. J. Primrose.

\section{Nicotinic Acid Tolerance Test}

SIR,-I read Drs. P. Ellinger and S. W. Hardwick's excellent paper (May 17, p. 672) and Capt. A. Erdei's letter (June 7, p. 822) with great interest. Both contributions suggest the intimate relationship of the functional state of the liver parenchyma to nicotinic acid metabolism, a problem elucidated mainly through the systematic work of Ellinger and collaborators in recent years. At the beginning of the war I had the opportunity of studying the physiology of the small intestine. I was able to submit evidence for the assumption that the gradient of absorption in the small intestine is to a great extent maintained by the catalysts of the intracellular respiratory enzyme systems. It is known that at least 3 water-soluble vitamins-thiamine, riboflavine, and nicotinic acid-are closely related to these catalysts, and it could be tentatively suggested that a deficiency in these vitamins and a liver "dysfunction" may be instrumental in the production of the sprue syndrome (Leitner, 1942). During subsequent years injections of crude liver extract (containing most of these enzymes) became a routine in the treatment of sprue. 